\title{
LES RECHERCHES SITOMÉTRIQUES AU POINT DE VUE DES REPEUPLEMENTS RATIONNELS
}

\author{
Par M. J.-A. LESTAGE (1) \\ Directeur de l'Aquarium de Bruxelles (Belgique).
}

Ce n'est un mystère pour nul d'entre nous que la question des repeuplements, notamment dans les eaux salmonicoles, fait actuellement lobjet de critiques pessimistes. Certains ont mème proclamé leur faillite.

Si nous consultons les auteurs qui traitent des causes du dépeuplement des eaux, nous voyons signalés comme facteurs prédominants : les pêches abusives, les pollutions, les barrages, les mauvais déversements, le hraconnage, les curages et faucardements mal compris, etc., etc. Il en est pourtant un autre qui est rarement mentionné, et dont tous ceux qui savent connaissent la primordiale importance; c'est la " déficience sitomótrique ".

L'amoindrissement et la disparition de tout ou partie du cheptel ichthyen ne sont la résultante ni de causes aveugles, ni d'un caprice du Poisson. Ils sont presque toujours concomitants à une modification biotopique qui retentit d'abord sur la faune nutritive, ensuite sur le Poisson.

Que le Poisson soit tributaire de la faune polymorphe qui hante le mềme milieu, ce n'est pas là une question bien neuve. Partout nous trouvons cette phrase stéréotypée : "Le Poisson se nourrit de Vers, de Larves, de Mollusques, etc. ". Mais, ce qui est relativement neuf, ce sont les recherches faites pour s'assurer si le biotope ichthyogène renferme une capacité biogénique adéquate et suffisante.

un a supprimé la formule simpliste d'antan

$$
\text { "Eau = Poisson " }
$$

pour lui substituer la formule plus scientifique

$$
\text { Eau }+ \text { nourriture }=\text { Poisson. }
$$

L'expérimentation de cette formule en a démontré la valeur.

Il fut un temps où tout ce qui n'était pas " plancton " semblait peu digne d'intérêt. Je me souviens de travaux où s'alignaient des listes immenses d'organismes planctoniques, et où les larves figuraient sous cette mention fort suggestive par son raccourci : - "larves diverses". Mais la raison en était peut-être que l'on possédait d'admirables monographies des êtres constituant le plancton, et aucun travail d'ensemble

(a) Communication destinée au VII ${ }^{e}$ Congrès international d'aquiculture et de peche, Paris $193 \mathrm{r}$. 
sur ces " larves diverses ". A combien de spécialistes planctonophiles a:-je montré pour la première fois une larve d'Ephéméroptère fort commune, celle du ciloeon!

Depuis qu'est née la limnoḷgie, et depuis, surtout, qu'elle a élargi son champ d'étude, restreint jadis aux lacs et étendu actuellement à toutes les eaux continentales, on a fini par voir quel immense intérèt il y avait à connaître, non plus " in vago ", mais d'une façon approfondie, tout ce monde jadis inconnu, ou connu seulement de rares spécialistes : - les phylobiontes et ceux qui sont secondairement phytophiles, - les pétricoles, - les limnophiles et les rhéophiles, - les curythermes et les sténothermes, etc.

On sait aujourd'hui ce que sont ces "larves diverses " qui sont mangées par le Poisson; - lesquelles sont utiles, indiflérentes ou nuisibles ; - lesquelles ont plus de valeur à cause de leur teneur en graisse, en protéine ou en azote. On sait aujourd'hui, par exemple, la valeur des formes cataloguées sous les noms de Trichoptères, Ephéméroptères, Plécóptères, etc..., et l'importance de leur dominante.

On sait aussi que, s'il y a parmi ces formes des types ubiquistes, la grande masse possède cependant une aecologie à ce point spécialisée que I'on peut absolument dire où les unes seront trouvées, où les autres ne le seront jamais. C'est pour avoir ignoré cette occologie que l'on a eu des surprises dans certains lacs artificiels, où l'on espérait que la faune rhéophile voisine viendrait en abondance peupler un biotope à facies limnétique.

On est donc en droit d'affirmer que la connaissance aussi parfaite que possible des composantes sitométrıques des biotopes ichthyogéniques doit ètre à la base de tout repeuplement rationnel, aussi bien pour l'alevin que pour l'adulte, aussi bien pour les types lénitiques que pour les iotiques.

Or, c'est au moment où se fondaient ces lois régénératrices que ia Z̈oologie officielle de certains pays nous a rendu le mauvais service de modifier son orientation. Nul n'ignore, en effet, que c'est la Biologie marine qui est en vogue aujourd'hui, et que ce serait une déchéance de s'occuper de l'Hydrobiologie.

A ceux qui pourraient croire que j'exagère, je conseille de dresser la liste comparative des Stations s'occupant de l'une cu de l'autre de ces Sciences. Ils peuvent aussi consulter maints récents traités de Zoologie, et voir le peu - avec souvent quelles erreurs - qui est réservé, par exemple, aux Insectes en général, aux groupes à lavies dulcicoles en particulier.

C'est donc en réagissant contre les tendances poussées à l'extrìme que l'on pourra lutter contre les pessimistes qui crient à la non-valeur des repeuplements, et qui pourraient finir par influencer ceux qui ne sont pas responsables de leur incompétence.

Tout comme l'étude des terrains précède leur aménagement agricole 
su forestier, c'est l'étude des biclupes qui doit précéder tout aménagement ichthyen. Par conséquent, il faut, pour certifier de la pauvreté ou de la rohesse d'un milieu ichthyogène, ne pas ètre incapable d'en dénombrer les composantes formant la capacité biogénique. Il faut, pour juger serieusement de l'action d'une pollution sur cette capacité biogénique, connaître quels organismes peurent en souffrir, et quelle répcrcussion peut se faire sentir sur leur cycle évolutif, et durant combien de temps, puisqu'il est certain qu'une eau rederenue pure n'est pas, pour cela, redevenue vivante.

On congoit que de telles notions ne doivent pas ètre livresques ou fractionnaires. Ces études doivent ètre l'ueuvre d'honmes rompus aux recherches de biologie lacustre, qui savent ce qu il taut chercher et ou il le faut chercher. Rien ne serail déplorable comme lamateurisme, car if $y$ aurait de grandes chances que les statistiques du potentiel nutritif seraient faussées et domeraient, par conséquent, une idée incomplète de la capacité biogénique réelle. De semblables erreurs pourraient coǹter cher.

C'est évidemment un raisonnenent analoģue qui a poussé tous les C.ongrès internationaux de pisciculture, convaincus de l'importance de la question, à rechercher les meilleurs moyens de fromouvoir cette investigation documentaire.

Tous se sont fait un point d'honneur de montrer le magnifique rôle des Stations de biologie lacustre, et nul n'ignore les résultats splendides qui ont été obtenus, dans les pays favorisés à cet égard, tant au point de vue scientifique et pédagogique qu'au point de vue économique

Certains pays, micux inspirés encore, n'ont pas hésité à adjoindre des laboratoires volants aux Stations parfois trop lointaines et handicapées do ce fait pour des études urgentes et à faire sur place.

Or, il est indéniable que c'est surtout aujourd'hui que s'imposent ces recherches de sitèse, aujourd'hui que la Nature recule davantage devant la Civilisation, aujourd'hui que le Poisson doit fuir de plus en plus devant l'usine qui monopolise son habitat, ces caux qui finiront par être l'élément fondamental non plus du cycle biologique du Poisson et des detres dont il se nourrissait, mais du cycle industriel.

Plus que jamais, donc, il nous faut des Stations réalisant ce triple pro:amme dont les données convergent en délinitive vers un but unique : le Poisson.

Programme scientifique. - Dresser l'inventaire qualitatif détaillé des productions végétales et animales, et aussi l'inventaire quantitatif de ces productions qui donnent à chaque biotope son facies particulier.

De ce programme découle un avancement certain de la Science par la description de faits nouveaux ayant trait à la systématique, à la dispersion géographique, à la biologie, à l'anatomie, à l'embryologie, etc., des formes dulcicoles.

Programme pédagogique. - Initier tous ceux que leur fonction met 
en rapport avec la question de l'aménagement des eaux aux notions au moins fondamentales, mais acquises pratiquement, sur le terrain même, nécessaires pour mener à bien cet aménagement. Ainsi saura-ton pratiquement quand une eau polluée, puis épurée, sera redevnue zoïque; si un endroit classique de déversement ronvient aussi par sa sitèse ; par conséquent, s'il le faut conserver ou modificr.

Ici šimposent les laboratoires volants, si en vogue en Imérigue par exemple.

Programme économique. - Transformer l'aquiculture et la pisciculture en branches rationuelles du savoir humain, et les dégager d'un empirisme qui a trop longlemps ven. Berhercher les causes scientifiques des dépeuplements et les moỵns scientifiques d'y remédier. Favoriser les recherches d'irhthyopathologie trop nécrigées parfois.

\title{
LES TRAVAUX DE LA STATION YOUGOSLAVE DE RECHERCHES CARPICOLES DURANT LES CINQ PREMIËRES ANNÉES
}

\author{
Par M. le Professeur Docteur E. RÖSSLER
}

do Mniversit: de Zagreb, Institut do \%oologic applique: (1).

Dès igıf. W. Zwrtave propriétaire d'étangs à Zdencina (Croatie). fit, aux autorités provinciales l'alors, l'offre de créer à ses frais, dans son domaine, une Station de recherehes carpicoles, pour fonctionner souts la direction de l'Idministration. Valheureusement, les rirconstances anormales qui prévalurent durant la guerre of les annés consécutives mirent obstacle à la réalisation de ce projet. I’installation des viviers of l'aménagement des lieux s'en trousèrent retardés. C'est seulement en rgoj, une fois surmontées des difficultés variées, que l'étahlissement, rattaché à l'Institut de zoologre applicués dont le siège est à Zagreh, put commencer ses Iravaux.

La Station dispose de six étangs de recherches d'une superficie grlobale de I Hectare 7 ares, six aulres ayant dû it re supprimés comme inutilisitbles. Ils sont situés au rentre du domaine, dans la vaste plaine au sud de la chaîne de Plejsivica, à environ in kilomètres de la gare de Zdencina, sır la ligne de Zagreb à Fiume.

Les viviers ont chacum leur alimentation séparée et empruntent leur eau

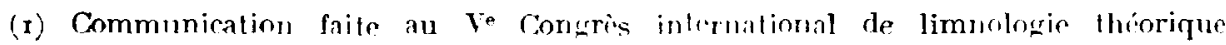
et appliquée, à liurlapest, en Aồt ı.30. 